\title{
CRESCIMENTO VEGETATIVO E PRODUTIVIDADE DE CAFEEIROS CONILON PROPAGADOS POR ESTACAS EM TUBETES
}

\author{
Vegetative growth and productivity of Conilon coffee-trees proceeding from seedlings \\ produced of deep-rooted cuttings in plastic tubes
}

\author{
José Augusto Teixeira do Amaral ${ }^{1}$, José Carlos Lopes ${ }^{1}$, José Francisco Teixeira do Amaral', \\ Sérgio Henriques Saraiva ${ }^{2}$, Waldir Cintra de Jesus $\mathbf{J r}^{1}$
}

\begin{abstract}
RESUMO
Objetivou-se neste trabalho avaliar o crescimento vegetativo e a produtividade de cafeeiros conilon (Coffea canephora Pierre ex Froehner), oriundos de mudas produzidas por estacas plantadas inicialmente em tubetes plásticos de $50 \mathrm{~cm}^{3}$ de capacidade. $\mathrm{O}$ experimento foi constituído de cinco tratamentos, que corresponderam aos tempos de permanência das estacas nos tubetes: $0 ; 15 ; 30$; 45 e 60 dias. Transcorridos esses tempos as mudas foram, sucessivamente, transplantadas para sacos de polietileno, contendo mistura de terra, esterco de curral e adubo químico, enviveiradas em um viveiro coberto com sombrite (50\%), provido de micro aspersão automática. As mudas permaneceram no viveiro com micro aspersão automática por 150 dias, quando então foram transferidas para o viveiro de aclimatação, onde ficaram por mais 30 dias. Após esse período, em setembro de 1999, as mudas foram plantadas em condições de campo, na área experimental do CCA-UFES, em Alegre, Sul do Estado do Espírito Santo. Foram feitas as seguintes medições: crescimento de ramos ortotrópicos e plagiotrópicos no segundo ano após o plantio e as quatro primeiras colheitas. A produção inicial de mudas de café conilon em tubetes não afetou o crescimento vegetativo, tampouco a produção de frutos.
\end{abstract}

Termos para indexação: Coffea canephora, kouillou, propagação vegetativa.

\section{ABSTRACT}

The objective of this work was to evaluate the vegetative growth and productivity of conilon coffee-tree (Coffea canephora Pierre ex Froehner), proceeding from seedlings produced of deep-rooted cuttings initially in plastic tubes with capacity of $50 \mathrm{~cm}^{3}$. The treatments were constituted of permanence period in plastic tube for $0 ; 15 ; 30 ; 45$ and 60 days. After these periods the plants were transplanted for polyethylene bags filled with substrate (soil + sand + manure bovine and chemical fertilization) maintained on the greenhouse with environment under shading canvas (50\%) and automatic micro aspersion during 150 days. After this time the plants were maintained during 30 days in acclimatization greenhouse management. Field experiment was set up in September of 1999, at experimental area CCA-UFES, in Alegre, south state of the Espírito Santo, Brasil. The following measurements were made: length of orthotropic and plagiotropic branches after first year and the first four productions. The results showed there were no differences either in vegetative growth and fruits production.

Index terms: Coffea canephora, kouillou, vegetative propagation.

(Recebido em 12 de dezembro de 2006 e aprovado em 8 de março de 2007)

\section{INTRODUÇÃO}

O Brasil é o maior produtor e exportador mundial de café desde meados do século XIX, quando a cafeicultura passou a ser uma atividade expressiva no mundo econômico (MATIELLO, 1998). O Estado do Espírito Santo é o segundo maior produtor de café do Brasil, sendo que o café conilon detém mais de $65 \%$ do seu parque cafeeiro (SILVA \& COSTA, 1995). O cultivo do cafeeiro conilon vem se expandindo no estado do Espírito Santo, nas regiões fisiograficamente baixas e de temperaturas relativamente elevadas (DADALTO \& BARBOSA, 1995), por se tratar de uma planta mais rústica, mais resistente à seca, a pragas e doenças, embora os padrões de tecnologia prioritários para a cultura sejam o plantio de mudas de estacas, podas corretivas, irrigação e nutrição equilibrada da lavoura (FERRÃO et al., 2004; MATIELLO, 1998).

O cafeeiro conilon é uma planta diplóide $(2 n=22$ cromossomos), apresentando auto-incompatibilidade gametofítica, sendo, portanto, auto-estéril e alógama (CARVALHO \& FAZUOLI, 1993; CONAGIN \& MENDES, 1961). Por conseguinte, as plantas dessa espécie, propagadas por sementes, não reproduzem as mesmas características genéticas desejáveis da planta matriz, podendo apresentar variações quanto à arquitetura da

'Doutores, Professores - Departamento de Produção Vegetal/DPV - Universidade Federal do Espírito Santo/UFES - Alto Universitário s/n - Cx. P. 16 29500-000 - Alegre, ES - jata@cca.ufes.br; jclopes@cca.ufes.br; jfamaral@cca.ufes.br; wcintra@cca.ufes.br

2Doutor - Departamento de Engenharia Rural/DER - Universidade Federal do Espírito Santo/UFES - Alto Universitário s/n - Cx. P. 16 - $29500-000$ Alegre, ES - sergio@cca.ufes.br 
planta, produtividade, resistência a doenças e pragas, época de maturação do fruto, tamanho e forma das sementes, dos frutos e das folhas. Por outro lado, a propagação vegetativa mantém as características genéticas da planta matriz, razão pela qual se utilizaram técnicas de propagação vegetativa por meio de estacas para essa espécie (BRAGANÇA et al., 1995; PAULINO et al., 1987).

O processo de produção de mudas de café em viveiros é feito em maior escala através de sacos de polietileno, contendo uma mistura de terra, esterco e adubo químico (BRAGANÇA et al., 1995; FERRÃO et al., 2004; PAULINO et al., 1987), que sob a ótica da praticidade, apresenta algumas desvantagens relacionadas às operações no viveiro, necessidade de maior disponibilidade de área física no viveiro e maior custo no transporte das mudas (CAMPINHOS JUNIOR \& IKEMORI, 1983). Assim, a busca de alternativas e inovações tecnológicas para redução no custo de produção tem conduzido à utilização de diversos recipientes para a produção de mudas. Embora haja carência de informações sobre resultados de condução de lavouras cafeeiras oriundas de mudas produzidas em tubetes, esta se pode apresentar como uma alternativa, pelas facilidades operacionais, como economia de espaço e mão-de-obra no viveiro de micro aspersão e facilidades para a seleção das mudas (MATIELLO, 1998), necessitando, entretanto, de maiores estudos sobre a viabilidade técnica de sua utilização. Além disso, as raízes crescem perpendiculares nos tubetes, não apresentando enovelamentos, sobretudo quando existem estrias longitudinais no interior dos tubetes e pela existência de um furo na extremidade inferior deles (GUIMARÃES et al., 1998).

Objetivou-se, com o experimento, avaliar o alongamento de ramos ortotrópicos e plagiotrópicos, o crescimento de área foliar e a produtividade de cafeeiros conilon, propagados por mudas formadas a partir de estacas, oriundas de brotos ortotrópicos, plantadas inicialmente em tubetes.

\section{MATERIAL E MÉTODOS}

Utilizaram-se cafeeiros conilon (Coffea canephora Pierre ex Froehner), clones precoces (Emcapa 8111), provenientes do Jardim Clonal de café conilon da Fazenda Experimental do CCA/UFES, localizada no município de Alegre-ES (Latitude Sul 20 45, 48,"; Longitude Oeste $41^{\circ} 31$ 57"; Altitude $250 \mathrm{~m}$ ).

Cinco meses antes de se iniciarem os trabalhos foram feitos vergamentos de ramos ortotrópicos nas plantas matrizes, para aumentar a brotação. $\mathrm{O}$ experimento teve início com a coleta dos brotos desses ramos, os quais foram acondicionados em sacos plásticos e transferidos para o local onde se prepararam as estacas, sempre mantendo os brotos umedecidos com água. Utilizaram-se mudas de nó inteiro, eliminando-se os ramos plagiotrópicos, por meio de poda, bem como metade das folhas de cada nó, sendo que o comprimento do entrenó basal foi de $4 \mathrm{~cm}$ e a extremidade basal do entrenó da estaca foi cortada em bisel, por meio de uma tesoura de poda (PAULINO et al., 1987). Fez-se a seguir o plantio das estacas e o enviveiramento em um viveiro recoberto com sombrite $(50 \%)$, provido de micro aspersão automática.

O experimento foi constituído de cinco tratamentos, que corresponderam aos tempos de permanência das estacas em tubetes: 0 (testemunha); 15; 30; 45 e 60 dias, contendo substrato Plantmax ${ }^{\circledR}$. As estacas testemunhas foram enviveiradas diretamente em sacos plásticos de polietileno. Os tubetes utilizados foram de polietileno, de cor escura, com capacidade de $50 \mathrm{~mL}$, contendo estrias longitudinais na face interior. Decorridos os tempos de permanência nos tubetes, as mudas clonais foram transferidas para sacos de polietileno, de $7 \mathrm{~cm}$ de diâmetro x $20 \mathrm{~cm}$ de altura e com perfurações na metade inferior. $\mathrm{O}$ substrato usado nos sacos de polietileno conteve uma mistura padrão de terra $\left(0,8 \mathrm{~m}^{3} \mathrm{~m}^{-3}\right.$ da mistura), esterco de curral $\left(0,2 \mathrm{~m}^{3} \mathrm{~m}^{-3}\right.$ da mistura) e adubo químico $(5 \mathrm{~kg}$ de

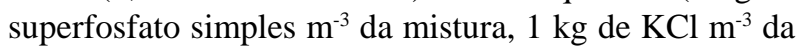
mistura e $5 \mathrm{~kg}$ de calcário $\mathrm{m}^{-3}$ da mistura).

As mudas permaneceram no viveiro com micro aspersão automática por 150 dias, quando então foram transferidas para o viveiro de aclimatação, onde permaneceram por 30 dias. Após esse tempo, as mudas foram plantadas no campo, em setembro de 1999, em covas de $40 \mathrm{~cm}$ x $40 \mathrm{~cm}$ x $40 \mathrm{~cm}$, num espaçamento de $2 \mathrm{~m}$ x $2 \mathrm{~m}$. O solo da área experimental é classificado como Latossolo Vermelho Amarelo Distrófico. A área experimental foi mantida no limpo por intermédio de capinas manuais. As adubações foram realizadas com base nas análises de solo, segundo as recomendações técnicas para a cultura (MATIELLO, 1998). O delineamento experimental foi o de blocos casualizados, com os cinco tratamentos e sete repetições, sendo três plantas por repetição. Os dados foram submetidos à análise de variância, utilizando o software estatístico SAEG (Sistema para Análises Estatísticas e Genéticas da Universidade Federal de Viçosa), versão 9.0 (EUCLYDES, 2004).

Em junho de 2000, no segundo ano após o plantio das mudas no campo, foram marcados 21 ramos ortotrópicos e 42 ramos plagiotrópicos, de cada tratamento, no terço superior da copa das plantas. O crescimento de área foliar foi determinado pelo método não destrutivo, 
com base no retângulo circunscrito no limbo foliar, medindo-se o maior comprimento e a maior largura, aplicando-se a fórmula de Awatramani \& Gopalakrishna (1965). Também foram feitos acompanhamentos sistemáticos do alongamento dos ramos ortotrópicos e plagiotrópicos pela técnica apresentada por Barros \& Maestri (1974).

As avaliações da produtividade das plantas de cada parcela, em cada tratamento, foram realizadas anualmente, a partir de 2001, nas quatro primeiras colheitas. Para tanto, os dados da produção em litros de café colhido por planta foram extrapolados para sacas de café beneficiadas de $60 \mathrm{~kg} \mathrm{ha}^{-1}$ (MATIELLO, 1998).

\section{RESULTADOS E DISCUSSÃO}

O crescimento de ramos e de folhas, plotado em termos de valores médios, por não existirem diferenças significativas entre os tratamentos, foi mínimo nos meses de junho, julho e agosto, bem como a partir da segunda quinzena de março (Figura 1). Essa variação no crescimento vegetativo do cafeeiro foi semelhante àquela anteriormente reportada por Libardi et al. (1998). As maiores taxas de crescimento de ramos e de folhas ocorreram em setembro, novembro e dezembro. Houve decréscimos temporários nas taxas de crescimento de ramos e de folhas durante a fase de crescimento ativo, na primeira quinzena de outubro e de dezembro. Também houve decréscimos no crescimento de janeiro a fevereiro, bem como a partir da segunda quinzena de março.
A periodicidade sazonal de crescimento vegetativo tem sido estudada por vários pesquisadores, tanto para o cafeeiro arábico (AMARAL et al., 2001, 2006; BARROS \& MAESTRI, 1974; DAMATTA et al., 1999), quanto para o conilon (LIBARDI et al., 1998), e várias as causas invocadas para explicar o fenômeno. De modo geral, o crescimento de folhas e de ramos ortotrópicos e plagiotrópicos (Figura 1) acompanhou as curvas de temperaturas mínima, média e máxima (Figura 2). Sabe-se que o intervalo de temperatura média anual ideal, para o cafeeiro conilon, situa-se entre 22 e $26^{\circ} \mathrm{C}$ (DADALTO \& BARBOSA, 1995). As maiores taxas de crescimento coincidiram com temperaturas mínimas acima de $17,5^{\circ} \mathrm{C}$, temperaturas médias situadas entre 22 e $26^{\circ} \mathrm{C}$ e temperaturas máximas em torno de $30^{\circ} \mathrm{C}$. Deve-se destacar que temperaturas máximas acima de $32^{\circ} \mathrm{C}$ estiveram correlacionadas com quedas acentuadas nas intensidades de crescimento, ocorridas durante a fase de crescimento ativo, na primeira quinzena de outubro e de dezembro, bem como da segunda semana de janeiro até a terceira semana de fevereiro, e na segunda quinzena de março de 2001. Possivelmente, a ocorrência de temperaturas do ar relativamente elevadas expliquem essas quedas no crescimento, porque folhas de café expostas a pleno sol podem apresentar temperaturas de 10 a $15^{\circ} \mathrm{C}$ acima da temperatura do ar (BUTLER, 1977). Nessas condições, essas elevações de temperatura em folhas de café fortemente iluminadas podem ocasionar decréscimos nas taxas fotossintéticas líquidas (NUTMAN, 1937).

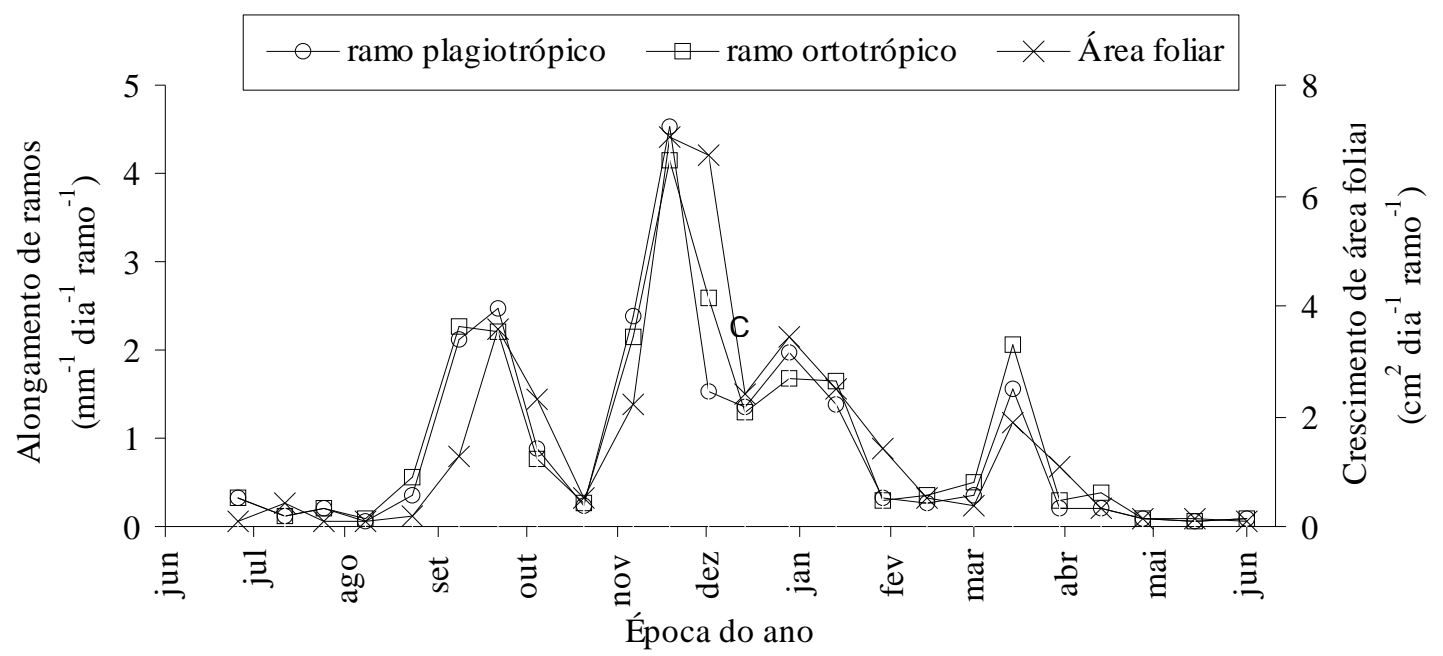

Figura 1 - Crescimento de ramos ortotrópicos e plagiotrópicos, e crescimento de área foliar de cafeeiros conilon de junho de 2000 a junho de 2001, UFES, Alegre - ES. 
Os decréscimos nas intensidades de crescimento ocorridos entre a segunda semana de janeiro e a terceira semana de fevereiro (Figura 1) podem ser atribuídos à fase de rápido enchimento dos grãos. Sabe-se que os frutos constituem os drenos mais fortes das plantas, podendo importar assimilados das folhas vizinhas e mesmo de regiões mais distantes na planta (CANNELL, 1970). Assim, cafeeiros com frutos apresentam menores intensidades de crescimento (AMARAL et al., 2001, 2006; LIBARDI et al., 1988).

O período de crescimento ativo (Figura 1) coincidiu, no tempo, com a época de maior precipitação pluvial (Figura 2), o inverso ocorrendo na fase de crescimento reduzido (Figura 1). Todavia, o crescimento declinou a partir de meados de março de 2001 (Figura 1), época em que choveu aproximadamente $40 \mathrm{~mm}$ (Figura 2). Além disso, choveu aproximadamente $60 \mathrm{~mm}$ no total dos meses de abril e maio (Figura 2), enquanto o crescimento estava praticamente paralisado (Figura 1). Esses resultados dão indicações de que a precipitação pluvial não explica a transição do estado de crescimento ativo ao estado de crescimento reduzido, e vice-versa, conforme sugerido anteriormente por outros pesquisadores (BARROS \& MAESTRI, 1974; BARROS et al., 1997; LIBARDI et al., 1988). Por outro lado, a queda temporária do crescimento de folhas e de ramos durante a primeira quinzena de dezembro (Figura 1) pode estar associada com maior precipitação, conforme sugerido por Sylvain (1958), quando registrou-se $240 \mathrm{~mm}$ (Figura 2).
Com relação à produção, também não houve diferenças significativas na produtividade das plantas em nenhuma das quatro colheitas (Tabela 1). A média das quatro primeiras colheitas foi inferior àquela observada por Ferrão et al. (2004), embora os dados de produção da quarta colheita deste trabalho fossem superiores à média de produção observada por aqueles pesquisadores. Considerando a capacidade das plantas de sustentarem uma alta carga pendente de frutos, pode-se inferir que o sistema radicular das mesmas apresenta-se bem desenvolvido, mesmo tendo sido as mudas produzidas inicialmente em recipientes relativamente pequenos.

A técnica de produção de mudas em tubetes tem provocado alguns problemas, sendo que, no norte do Estado, muitos cafeeiros tiveram morte precoce, quando foram diagnosticados problemas de má formação no sistema radicular, em decorrência do plantio de mudas provenientes de tubetes (CAFÉ..., 1998). O sistema de produção de mudas em tubetes necessita de cuidados e habilidade nas operações subseqüentes de repicagem das mudas dos tubetes para as sacolas ou diretamente dos tubetes para o campo (MATIELLO, 1998), evitando-se problemas em decorrência da má conformação do sistema radicular. Sabe-se que, nos tubetes, as raízes crescem perpendiculares, sobretudo quando existem estrias longitudinais no seu interior e em virtude da existência de um furo na sua extremidade inferior (GUIMARÃES et al.,

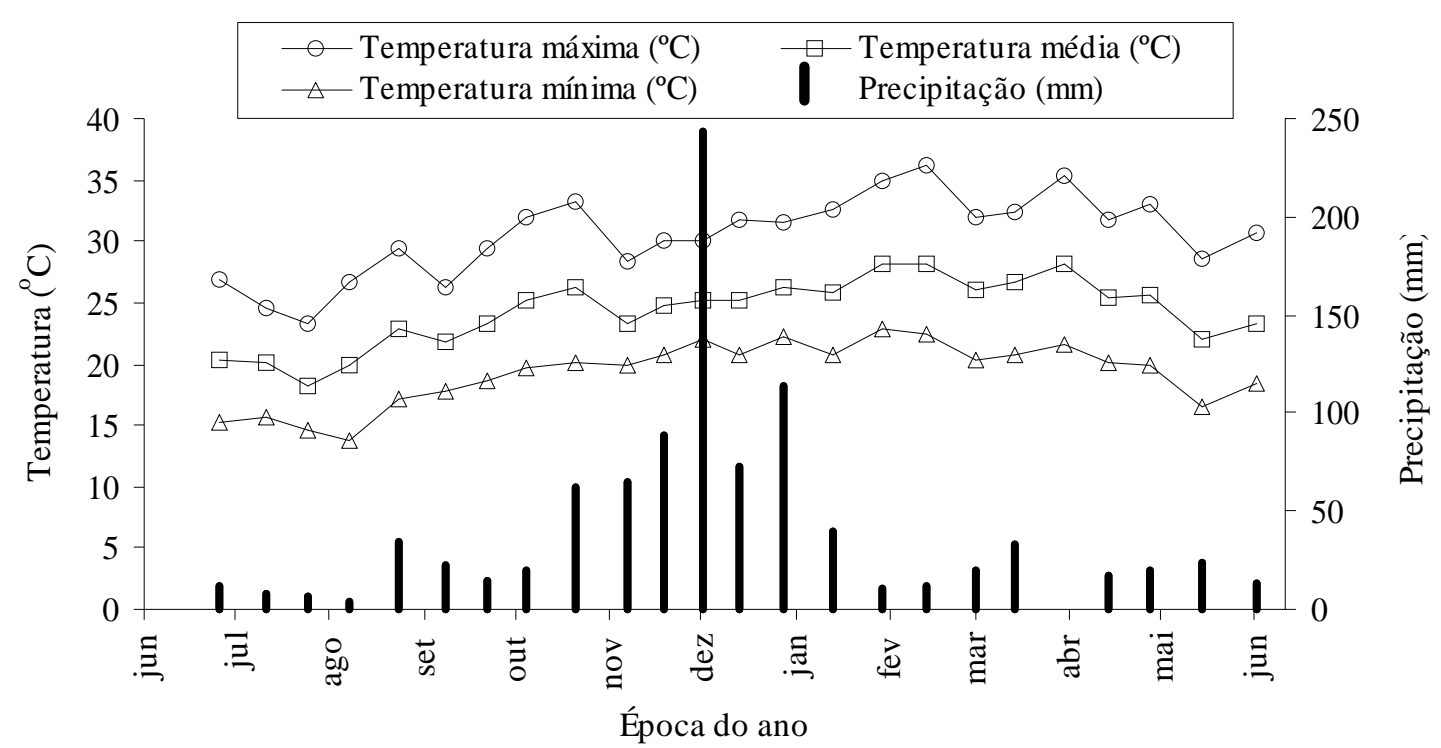

Figura 2 - Média das temperaturas máxima, média e mínima do ar, e precipitação pluvial de junho de 2000 a junho de 2001, UFES, Alegre - ES. 
Tabela 1 - Produção média das quatro primeiras colheitas de cafeeiros conilon, propagados por mudas formadas a partir de estacas, plantadas inicialmente em tubetes, transplantadas após os períodos de 0; 15; 30; 45 e 60 dias para sacolas de polietileno, UFES, Alegre - ES.

\begin{tabular}{|c|c|c|c|c|}
\hline \multirow{2}{*}{$\begin{array}{c}\text { Períodos das estacas } \\
\text { em tubetes (dias) }\end{array}$} & \multicolumn{4}{|c|}{ Colheitas } \\
\hline & Primeira & Segunda & Terceira & Quarta \\
\hline & \multicolumn{4}{|c|}{ Sacas de café beneficiadas de $60 \mathrm{~kg} \cdot \mathrm{ha}^{-1}$} \\
\hline 0 & 11,02 & 16,25 & 36,02 & 67,66 \\
\hline 15 & 12,50 & 15,78 & 34,14 & 60,23 \\
\hline 30 & 10,78 & 17,27 & 42,19 & 63,59 \\
\hline 45 & 10,94 & 15,00 & 38,67 & 67,34 \\
\hline 60 & 10,47 & 16,80 & 32,34 & 60,63 \\
\hline Médias & 11,14 & 16,22 & 36,67 & 63,89 \\
\hline $\mathrm{CV}(\%)$ & 38,06 & 35,81 & 29,67 & 19,51 \\
\hline
\end{tabular}

1998). Outra vantagem do uso de tubetes refere-se ao substrato, pois além das muitas possibilidades disponíveis, os substratos artificiais têm sido uma opção pelas facilidades no transporte e no enchimento dos tubetes, e como são compostos inertes, apresentam segurança contra infestação de nematóides e permitem o desenvolvimento das mudas quando acrescidos de adubo químico (GUIMARÃES et al., 1998).

\section{CONCLUSÃO}

A formação de mudas de café conilon, inicialmente em tubetes, não afeta o crescimento vegetativo nem a produtividade.

\section{REFERÊNCIAS BIBLIOGRÁFICAS}

AMARAL, J. A. T. do; DAMATTA, F. M.; RENA, A. B. Effects of fruiting on the growth of arabica coffee trees as related to carbohydrate and nitrogen status and to nitrate reductase activity. Revista Brasileira de Fisiologia Vegetal, Lavras, v. 13, n. 1, p. 66-74, abr. 2001.

AMARAL, J. A. T. do; RENA, A. B.; AMARAL, J. F. T. do. Crescimento vegetativo sazonal do cafeeiro e sua relação com fotoperíodo, frutificação, resistência estomática e fotossíntese. Pesquisa Agropecuária Brasileira, Brasília, v. 41, n. 3, p. 377-384, mar. 2006.
AWATRAMANI, N. A.; GOPALAKRISHNA, H. K. Measurement of leaf area in coffee: II. Coffea robusta. Indian Coffee, Chikmagalur, p. 10-12, June 1965.

BARROS, R. S.; MAESTRI, M. Influência dos fatores climáticos sobre a periodicidade de crescimento vegetativo do café (Coffea arabica L.). Revista Ceres, Viçosa, v. 21, p. 268-279, jul./ago. 1974.

BARROS, R.; MOTA, J. W. S.; DAMATTA, F. M.; MAESTRI, M. Decline of vegetative growth in Coffea arabica L. in relation to leaf temperature, water potential and stomatal conductance. Field Crops Research, Madison, v. 54, n. 1, p. 65-72, Aug. 1997.

BRAGANÇA, S. M.; FONSECA, A. F. A.; SARAIVA, J. S. T.; PEREIRA, J. O.; ROCHA, A. C.; PELISSARI, S. A.; BREGONCI, I. S. Formação de mudas. In:__. Manual técnico para a cultura do café no Estado do Espírito Santo. Vitória: Secretaria de Estado de Agricultura, 1995. p. 19-28.

BUTLER, D. R. Coffee leaf temperatures in a tropical environment. Acta Botanica Neerlandica, Amsterdam, v. 26, n. 2, p. 129-140, Apr. 1977.

CAFÉ clonal provoca prejuízo no Norte. A Gazeta, Vitória, ES, 9 ago. 1998. 
CAMPINHOS JÚNIOR, E.; IKEMORI, Y. K. Introdução de nova técnica na produção de mudas de essências florestais. Silvicultura, São Paulo, v. 8, n. 28, p. 226-228, jan./fev. 1983.

CANNELL, M. G. R. The contribution of carbohydrates from vegetative laterals of the growth of fruits on the bearing branches of Coffea arabica. Turrialba, San José, v. 20, n. 1, p. 15-19, ene./mar. 1970.

CARVALHO, A.; FAZUOLI, L. C. Café. In: FURLANI, A. M. C.; VIÉGAS, G. P.O melhoramento de plantas no instituto agronômico. Campinas: Instituto Agronômico, 1993. v. 1, p. 29-76.

CONAGIN, C. H. T. M.; MENDES, A. J. T. Pesquisas citológicas e genéticas em três espécies de Coffea: autoincompatibilidade em Coffea canephora Pierre ex Froehner. Bragantia, Campinas, v. 20, n. 34, ago. 1961.

DADALTO, G. G.; BARBOSA, C. A. Macrozoneamento agroecológico. In: ___ Manual técnico para a cultura do café no Estado do Espírito Santo. Vitória: Secretaria de Estado de Agricultura, 1995. p. 11-14.

DAMATTA, F. M.; AMARAL, J. A. T.; RENA, A. B. Growth periodicity in trees of Coffea arabica $\mathrm{L}$. in relation to nitrogen supply and nitrate reductase activity. Field Crops Research, Madison, v. 60, n. 3, p. 223-229, Feb. 1999.

EUCLYDES, R. F. Sistema para análises estatísticas e genéticas. Versão 9.0. Viçosa: FUNARBE/UFV, 2004.

FERRÃO, R. G.; FONSECA, A. F. A. da; FERRÃO, M. A. G.; MUNER, L. H. de; VERDIN FILHO, A. C.; VOLPI, P. S.; MARQUES, E. M. G.; ZUCATELI, F. Café conilon: técnicas de produção com variedades melhoradas. 2. ed. Vitória: Incaper, 2004. $60 \mathrm{p}$.

GUIMARÃES, P. T. G.; ANDRADE NETO, A.; BELLINI JÚNIOR, O.; ADÃO, W. A.; SILVA, E. M. A produção de mudas de cafeeiros em tubetes. Informe Agropecuário, Belo Horizonte, v. 19, n. 193, p. 98-109, maio/jun. 1998.

LIBARDI, V. C. de M.; AMARAL, J. A. T. do; AMARAL, J. F. T. do. Crescimento vegetativo sazonal do cafeeiro (Coffea canephora Pierre var. conilon) no Sul do Estado do Espírito Santo. Revista Brasileira de Agrometeorologia, Santa Maria, v. 6, n. 1, p. 23-28, jan./jun. 1988.

MATIELLO, J. B. Café conilon: como plantar, tratar, colher, preparar e vender. Rio de Janeiro: MM Produções Gráficas, 1998. $162 \mathrm{p}$.

NUTMAN, F. J. Studies of the physiology of Coffea arabica: I. photosynthesis of coffee leaves under natural conditions. Annals of Botany, Oxford, v. 1, n. 3, p. 353-367, July 1937.

PAUlinO, A. J.; MATIELlO, J. B.; PAULINI, A. E.; BRAGANÇA, J. B. Cultura do café conillon: instruções técnicas sobre a cultura do café no Brasil. Rio de Janeiro: MIC-IBC-DIPRO, 1987. 43 p.

SILVA, A. E. S.; COSTA, E. B. Importância econômica e social. In: _. Manual técnico para a cultura do café no Estado do Espírito Santo. Vitória: Secretaria de Estado de Agricultura, 1995. p. 9-10.

SYLVAIN, P. G. El ciclo de crescimiento de Coffea arabica. Turrialba: Instituto Interamericano de Ciências Agrícolas, 1958. $17 \mathrm{p}$. 